\title{
The Next Generation of NICU Staff
}

Kelly Welton, RRT-NPS

2020 wreaked havoc on all of us. Life's rhythm as we knew it in 2019 got transformed as we learned a new way to do almost everything in 2020. Although Zoom and Amazon saved many people from unnecessary trips to the office and the store, one thing remained unchangeable: healthcare, specifically patient care, is a hands-on business.

Since patient care is a hands-on profession, training requires a mentor to show us the how-tos. It is one thing to learn how to set up a ventilator or an IV pump; another thing entirely to troubleshoot one that's not doing what you set it to do.

Thanks to SARS-Covid-19 [CoVid], many respiratory therapy and nursing school programs closed. First, clinical sites closed their doors so as not to expose students to this new virus that was spreading fast. Then schools closed their doors to in-person instruction. Forced to learn online, recent graduating classes will only get their 'hands-on 'experience once hired.

\section{"In Southern California, there were two programs available that provided in-depth NICU classes and clinical training. Both programs have subsequently closed, leaving nowhere for therapists to get basic NICU training."}

In the hospitals, current staff who want to be trained to work in NICU often need approval from their manager. In Southern California, there were two programs available that provided in-depth NICU classes and clinical training. Both programs have subsequently closed, leaving nowhere for therapists to get basic NICU training. Managers are also tasked with being chronically shortstaffed, unable to let a staff member shadow a NICU RT for a day because they are desperately needed in the other hospital areas. This situation has been true for decades; however, CoVid demanded all-hands-on-deck, and there was no time for any RT not to carry a workload.

As an RT Educator, my role has been to find the areas in which staff need additional training. Whether it is low-use equipment or training in a new area, competency in not just knowledge. Critical thinking and troubleshooting are also 'musts.' Although the NICU was not left untouched by Covid, when the CoVid dust settles, many RT's and RN's will either leave the field or seek solace in a place that is not calling a code every hour and losing 3-4 patients per shift. When these seasoned therapists retire, who will be left to care for patients? For these reasons, the Academy of Neonatal Care was formed. Initially, AoNC was designed to be a hands-on workshop. Participants learn the foundation of neonatal respiratory care and participate in workshops practicing correct fitting of nasal prongs, changing Oscillator circuits, surfactant instillation, and more. Covid has now challenged AoNC as well to translate learning to an online format. Clinical competency software and the ability to present live online courses where students can ask questions in real-time help AoNC fill the void.

\section{"As a non-profit 501 (c) 3, the Academy of Neonatal Care's goal is to teach the highest level of care to beginners in NICU and seasoned therapists. RN's are welcome to join, as are physicians."}

As a non-profit 501 (c) 3, the Academy of Neonatal Care's goal is to teach the highest level of care to beginners in NICU and seasoned therapists. RN's are welcome to join, as are physicians.

As a non-profit, the Academy of Neonatal Care's goal is to teach the highest level of care to beginners in NICU and seasoned therapists. RN's are welcome to join us, as are physicians.

Our secondary goal is to reach out to NICU babies' parents and family to support them while their baby is in our care. Lastly, as a non-profit, we will give back by contributing to community outreach and support healthy pregnancy and healthy baby efforts across the world.

With the release of a CoVid vaccine, we look forward to presenting live and in -person. AoNC's platform continues to change with the times, but our 'base camp' remains the same. We built day one for beginner RT's and RN's who have wanted to learn NICU but have never been given a chance to get into a NICU with a preceptor.

Day 2 is designed for the RT that floats to NICU occasionally and wants a refresher to reinforce skills and knowledge.

Day 3 is designed for current NICU staff who want to expand their knowledge on subjects such as Jet ventilation, iNO, Transports,

NEONATOLOGY TODAY is interested in publishing manuscripts from Neonatologists, Fellows, NNPs and those involved in caring for neonates on case studies, research results, hospital news, meeting announcements, and other pertinent topics.

Please submit your manuscript to: LomaLindaPublishingCompany@gmail.com 
and more. The way we are going, we may soon have a full 5-day course!

\section{"That certificate carries significant weight} with the participant's employer or director, enough so that the manager would feel confidant pairing the RT with a mentor to help them assimilate into NICU."

Academy of Neonatal Care's vision is that when the first three days are completed, the RT has a certificate from AoNC that says, "I finished the entire AoNC course, and am now ready to work with a preceptor." That certificate carries significant weight with the participant's employer or director, enough so that the manager would feel confidant pairing the RT with a mentor to help them assimilate into NICU.

Disclosure Statement: The author has no relevant conflicts of interest to declare.

NT

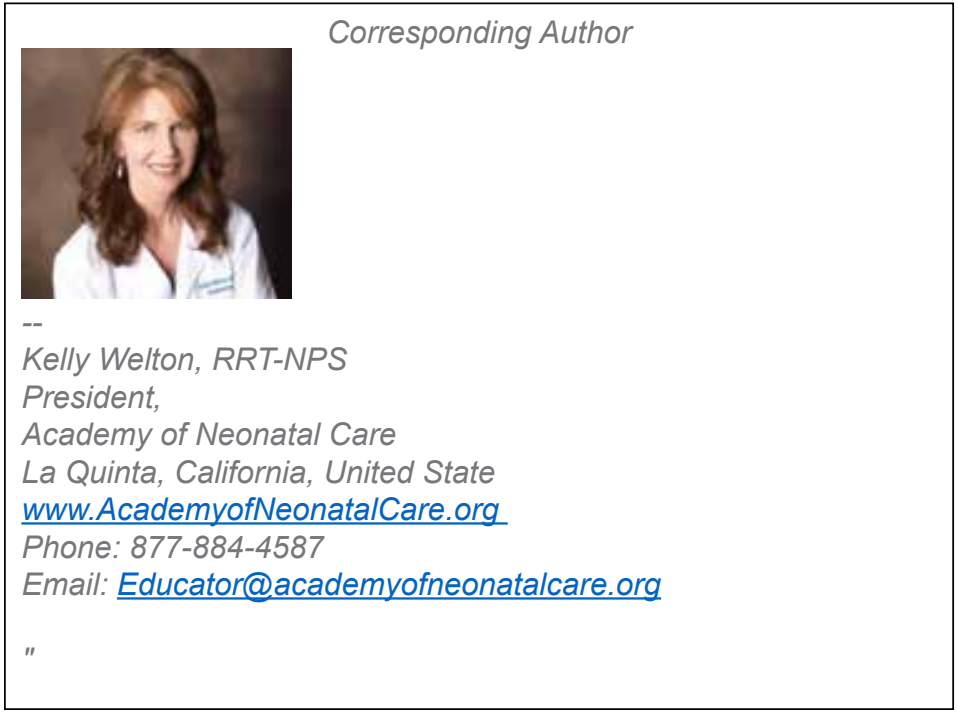

Readers can also follow NEONATOLOGY TODAY via our Twitter Feed @NEOTODAY

\section{embrace:

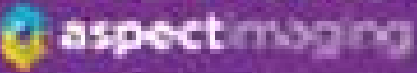
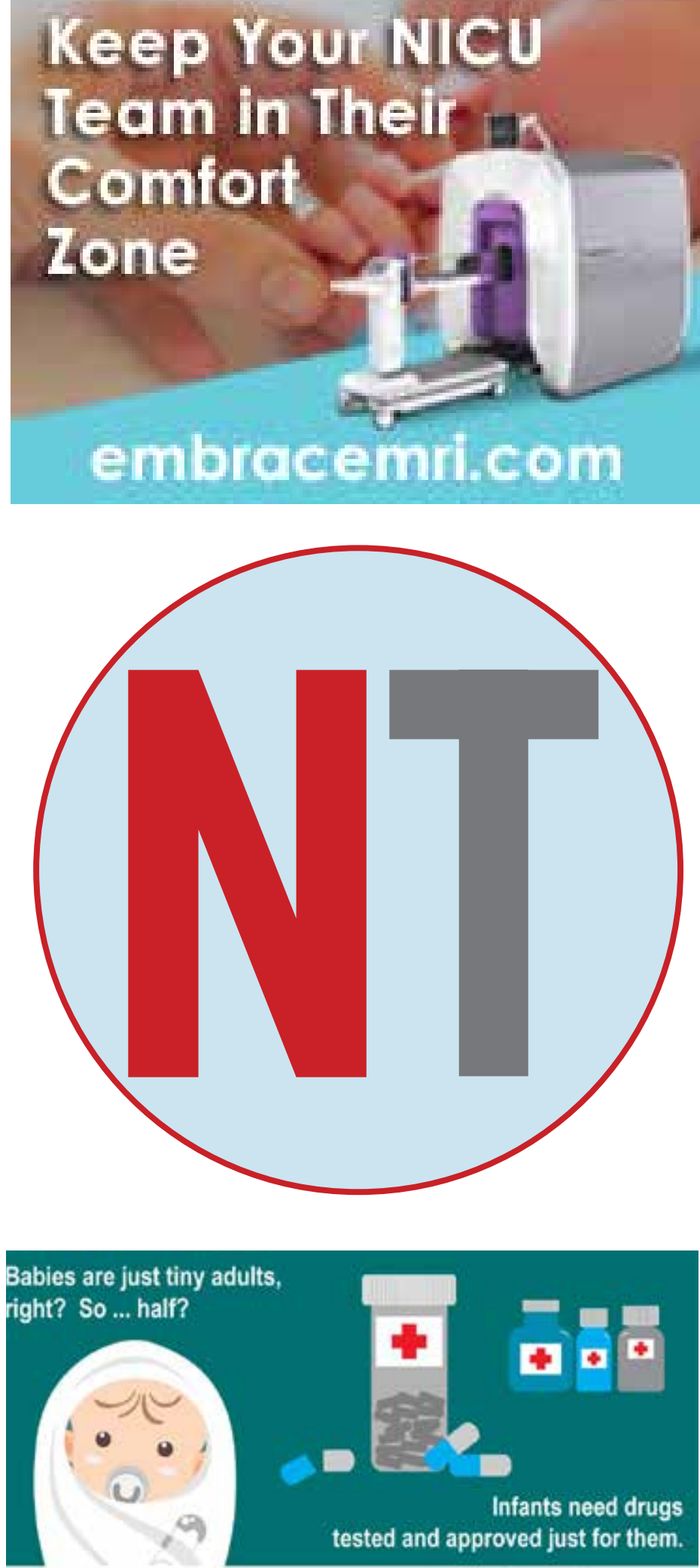